\title{
Physische Attraktivität und individuelles Leistungsverhalten
}

\author{
oder: warum und wann unattraktive Männer die besseren Fußballer sind
}

\author{
Von Ulrich Rosar, Jörg Hagenah und Markus Klein
}

Zusammenfassung: Dass attraktive Menschen im Berufsleben mehr Erfolg haben als unattraktive Menschen, ist in der sozialpsychologischen Literatur breit dokumentiert. Hingegen ist weitgehend unerforscht geblieben, ob attraktive Menschen zur Erzielung dieses Erfolgs auch eine höhere Leistung erbringen. Da attraktive Menschen von ihrer sozialen Umwelt besser behandelt werden, können sie ihren höheren Erfolg möglicherweise auch mit geringeren Anstrengungen erzielt haben. Es stellt sich also die Frage, wie sich die Attraktivität eines Menschen auf seine Leistung auswirkt. Da das Leistungsverhalten von Profifußballern relativ einfach feststellbar und außerdem vergleichsweise gut dokumentiert ist, wird dieser Frage am Beispiel der 483 Fußballprofis nachgegangen, die in der Saison 2007/2008 bei den 18 Mannschaften der 1. Bundesliga zum Einsatz kamen. Die Attraktivität der Spieler wird dabei gemäß der Truth of Consensus Method durch Mittelwertbildung über die im Rahmen einer Online-Umfrage - auf der Grundlage von Portraitphotographien erhobenen Attraktivitätsurteile erfasst. Das Leistungsvermögen der Spieler wird über die detaillierte Beobachtung ihres Spielverhaltens gemessen. In der statistischen Auswertung zeigt sich, dass die Attraktivität der Spieler ihre Leistungsfähigkeit tendenziell negativ beeinflusst. Der Effekt hängt allerdings von der Attraktivität der Spieler in der jeweiligen Mannschaft ab: Ist die Attraktivität aller Spieler hoch und homogen, dann kehrt sich die Richtung des Effekts der Attraktivität auf das Leistungsverhalten um.

\author{
„Ball is rund, Spiel dauert 90 Minuten. Soviel is \\ schoma klar. - Alles andere is Theorie. "
}

Der Wachmann Schuster (Armin Rohde) in der Eröffnungsszene des Films Lola rennt von Tom Tykwer

\section{Einleitung: Physische Attraktivität wirkt - aber wie wirkt sie auf individuelles Leistungsverhalten?}

Aus dem oben angeführten Filmzitat spricht eine tiefe Einsicht in die Unvorhersagbarkeit menschlicher Entscheidungen und Folgen sozialen Handelns in komplexen Interaktionsprozessen. Wer den Film Lola rennt kennt, weiß, dass das Thema Nummer 1 deutscher Männer hier als Metapher dient. Der Verweis auf die Situation des Fußballspiels soll verdeutlichen, dass bereits kleine Abweichungen im Handeln sozialer Akteure und geringfügige Variationen der Kontextbedingungen, unter denen die Handlungen vollzogen werden, zu völlig disparaten Ergebnissen führen können. Das Fußballspiel bildet dies en miniature ab. Aus der Makroperspektive betrachtet hängt der Verlauf eines Spiels von den Leistungen der Spieler, dem Zusammenspiel der Mannschaften, den taktischen Vorgaben der Trainer und der Unterstützung der Fans ab. Genauso spielen aber auch die Aktionen und Reaktionen der Gegner, die Entscheidungen der Schiedsrichter oder verletzungsbedingte Ausfälle eine Rolle. Und selbst die Platzverhältnisse und die Frage, wer zuerst in Führung geht, können den Spielausgang entscheidend beeinflussen. Aus der Mikroperspektive des einzelnen Spielers betrachtet, entscheiden nicht nur die eigene Leistungsfähigkeit und die eigene Leistungsmotivation über den spielerischen Erfolg. Hier sind ebenso die Erwartungen und Entscheidungen der anderen Akteure und die Rahmenbedingungen, unter denen ein Spieler agieren muss, von Bedeutung. Insofern ist die Situation des Fußballspiels nicht nur ein geeignetes Sinnbild für komplexe Handlungssituationen. Sie ist auch ein geeignetes Untersuchungsfeld, um soziologisch relevante Frage- 
stellungen empirisch analysieren zu können (vgl. exemplarisch Berger / Hammer 2007; Esser 1991; Kalter 1999; 2005).

Wir haben das Filmzitat unserer Abhandlung aber nicht nur deshalb vorangestellt, weil es die Komplexität von Fußballspielen versinnbildlicht und eine Konjunktion zwischen der Sphäre des Fußballs und den profanen Handlungssituationen des Alltags herstellt. Wir haben es auch ausgewählt, weil es als Quintessenz eines analytischen Dilemmas verstanden werden kann, das dank der besonderen Datenlage am ehesten anhand des deutschen Profifußballs einer empirischen Klärung zugeführt werden kann. Die Attraktivitätsforschung hat für eine Vielzahl von Lebensbereichen und nahezu über alle Lebensphasen hinweg nachgewiesen, dass die äußere Anmutung eines Menschen seine Chancen auf sozialen Erfolg beeinflusst. Auch wenn die soziologische Forschung - und hier insbesondere die deutschsprachige soziologische Forschung - erst in jüngerer Zeit (wieder)entdeckt ${ }^{1}$, dass die physische Attraktivität eines Menschen analog zum Geschlecht oder der Ethnie ein bedeutsamer Prädiktor sozialer Ungleichheit und Diskriminierung ist, so steht ihre grundsätzliche soziale Relevanz dennoch außer Frage. Ihre Wirksamkeit ist durch empirische Forschungsarbeiten gut dokumentiert, die zwar vorwiegend sozialpsychologisch ausgerichtet sind, gleichwohl aber in die Hunderte gehen (vgl. zur Übersicht Grammer 2002; Renz 2006). Als Zusammenfassung dieser Forschungsarbeiten lassen sich fünf zentrale Mechanismen identifizieren, über die die physische Attraktivität ihre Wirkung entfaltet. Dies sind der Attractiveness Consensus, der Attractiveness Attention Boost, das Attractiveness Stereotype, der Attractiveness Treatment Advantage und der Attractiveness Glamour Effect (Klein / Rosar 2006: 307 f; Rosar et al. 2008: 67). Sie sorgen dafür, dass schöne Menschen gegenüber ihren weniger ansehnlichen Zeitgenossen - selbst unter Kontrolle objektiver Leistungsmerkmale - einen Attractiveness Competition Advantage haben (Rosar et al. 2008: 67). Wendet man die Mechanismen aber auf individuelles Leistungsverhalten an, so lassen sich aus ihnen zwei Schlussfolgerungen für den Zusammenhang zwischen physischer Attraktivität und objektiver Leistung ableiten, die einander diametral entgegengesetzt sind, hypothetisch jedoch dieselbe Evidenz beanspruchen dürfen. Logisch lässt sich dieser Widerspruch nicht auflösen. Er bedarf der empirischen Klärung.

Mit der in diesem Aufsatz vorgestellten Untersuchung wurde der Versuch einer solchen empirischen Klärung unternommen. Der Aufsatz gliedert sich im weiteren Fortgang in vier Schritte. Zunächst werden wir kurz die Mechanismen skizzieren, über die die physische Attraktivität ihre Wirkung entfaltet, diese Mechanismen für die Handlungslogik des Fußballplatzes adaptieren und die beiden widersprüchlichen Schlussfolgerungen zum individuellen Leistungsverhalten attraktiver und unattraktiver Profifußballer herausarbeiten (Abschnitt 2). Nach einer Beschreibung der Datenbasis (Abschnitt 3) folgt die Darstellung der empirischen Analysen (Abschnitt 4). Den Abschluss bilden eine Zusammenfassung der wesentlichen Befunde und einige weiterführende Schlussfolgerungen (Abschnitt 5).

\section{Theoretische Erwägungen: Physische Attraktivität und fußballerische Leistung}

Die bisher vorliegenden Studien der Attraktivitätsforschung decken ein weites Feld soziologisch relevanter Arbeitsgebiete ab. Das Spektrum reicht hier von Partnermärkten über Gütermärkte bis hin zu Arbeitsmärkten (Benoy 1982; DeShields et al. 1996; Elder 1969; Feingold 1988; Gangestad / Thornhill 1997; Kanazawa / Still 2000; Langlois et al. 2000; Marwick 1988; Reingen / Kernan 1993; Shackelford et al. 2000; Solnick / Schweitzer 1999; Thornhill / Gan-

1 In diesem Zusammenhang ist es fast schon eine ironische Fußnote der Wissenschaftsgeschichte, dass die nach modernem Wissenschaftsverständnis erste fundierte empirische Studie zur sozialen Wirkung der physischen Attraktivität von dem U.S.-amerikanischen Soziologen Willard W. Waller (1937) durchgeführt und in einem der heute führenden soziologischen Journals publiziert wurde. 
gestad 1994; Thornhill et al. 1995; Udry / Eckland 1984; Walster et al. 1966; White 1980). Analysen zu Karrierechancen lassen sich ebenso finden wie Untersuchungen zu Kindergartenfreundschaften (Anderson et al. 2001; Biddle / Hamermesh 1998; Cash et al. 1977 a; Dion / Berscheid 1974; Hamermesh / Biddle 1994; Hanson Frieze et al. 1991; Harper 2000; Hosoda et al. 2003; Krantz 1987; Mazur et al. 1984; Mueller / Mazur 1996; Rost 1993; Vaughn / Langlois 1983). Universelle lebenszyklische Phasen, wie beispielsweise die Pubertät, sind genauso vertreten wie die außeralltägliche Grenzsituation einer psychischen Erkrankung (Cash et al. 1977 b; Crouch / Degelman 1998; Guerra 1998; Lerner et al. 1990; Rosenblum / Lewis 1999; Savin-Williams 1979; Turner et al. 1997). Und der Fokus der Untersuchungen richtet sich mit derselben Selbstverständlichkeit auf Säuglinge und Schüler wie auf Straftäter und Spitzenpolitiker (Baugh / Parry 1991; Budesheim / DePaula 1994; Clifford / Walster 1973; Deseran / Chung 1979; Dion et al. 1972; Downs / Lyons 1991; Efran 1974; Esses / Webster 1988; Klein / Rosar 2007; 2009; Landy / Sigall 1974; Langlois et al. 1995; 2000; Mace 1972; Maisonneuve / Bruchon-Schweitzer 1999; Rosar 2009; Ross / Salvia 1975; Rost 1993; Steffensmeier / Terry 1973). Die physische Attraktivität scheint in ihrer Wirkung geradezu ubiquitär zu sein und es lässt sich kaum ein Feld soziologischer Ungleichheitsforschung ausmachen, auf dem die Attraktivitätsforschung nicht in der einen oder anderen Weise nachgewiesen hätte, dass die äußere Anmutung eines Menschen seine Chancen auf sozialen Erfolg beeinflusst.

Alle Studien der Attraktivitätsforschung starten dabei von der Erkenntnis, dass die Schönheit eines Menschen nicht so sehr im Auge des Betrachters liegt, sondern vor allem ein objektives Merkmal der betrachteten Person ist (Köhler 1984: 140ff). Differenzen in der Attraktivitätsbeurteilung durch verschiedene Betrachter sind in der Regel marginal und gehen auf nachrangige Geschmacksunterschiede zurück. Insgesamt überwiegt die Übereinstimmung in den Urteilen unterschiedlicher Betrachter über die physische Attraktivität einer konkreten Person. Dieses Phänomen bezeichnet man als den Attractiveness Consensus (Cunningham 1986; Cunningham et al. 1990; 1995; Henss 1987; 1992; Iliffe 1960). Auf ihn bauen die klar beschreibbaren Wirkungen der physischen Attraktivität auf. An erster Stelle steht hier der Attractiveness Attention Boost. Attraktiven Menschen wird von ihrer Umwelt mehr Aufmerksamkeit entgegengebracht (Maner et al. 2003). Sie werden dabei nicht nur eher und intensiver wahrgenommen, ihre Aussagen und Handlungen werden auch besser von den Betrachtern memoriert. Daneben profitieren schöne Menschen davon, dass ihnen eine ganze Reihe positiv bewerteter Persönlichkeitseigenschaften zugeschrieben werden (Dermer / Thiel 1975; Dion et al. 1972; Eagly et al. 1991; Feingold 1992; Miller 1970). Dank des Attractiveness Stereotype gelten sie ihren Mitmenschen beispielsweise als leistungsfähiger, fleißiger, intelligenter, durchsetzungsfähiger, kreativer und sozial verträglicher. Selbst ein objektives Fehlverhalten ihrerseits muss dieses positive Image nicht zwangsläufig beschädigen. In solchen Fällen dürfen attraktive Menschen auf eine ausgeprägte Neigung ihrer Zeitgenossen bauen, das Fehlverhalten zu relativieren oder die Ursachen äußeren Umständen zuzuschreiben, die von der handelnden Person nicht zu verantworten sind. Dies ist der Attractiveness Glamour Effect (Bassili 1981; Dion et al. 1972; Grammer 2002: 169). In konkreten Interaktionssituationen profitieren schöne Menschen zudem vom Attractiveness Treatment Advantage: Ihre Interaktionspartner begegnen ihnen nachweislich mit mehr Respekt und Hochachtung, sie erfahren mehr Hilfe und Unterstützung durch ihr soziales Umfeld und sie genießen grundsätzlich eine höflichere und zuvorkommendere Behandlung durch andere (Benson et al. 1976; Dabbs / Stokes 1975; Dion / Berscheid 1974; Hartnet et al. 1974; Hatfield / Sprecher 1986; Langlois et al. 2000; Marwick 1988; McCabe 1988; Mulford et al. 1998; Ritter et al. 1991; White Stephan / Langlois 1984). Zusammengenommen führen diese Mechanismen dazu, dass attraktive Menschen gegenüber ihren weniger ansehnlichen Zeitgenossen klar im Vorteil sind - sie genießen einen Attractiveness Competition Advantage. 
Überträgt man die Mechanismen auf Profifußballer und ihre sportlichen Leistungen, so kann zunächst einmal davon ausgegangen werden, dass attraktive Fußballspieler in der Lage sein sollten, bessere Leistungen zu erbringen. Dank des Aufmerksamkeitseffekts werden ihre Mitspieler sie im Verlauf eines Fußballspiels häufiger und intensiver beobachten, ihre Absichten eher verstehen und so besser in der Lage sein, das eigene Spiel an diesen Absichten auszurichten. Zudem ist davon auszugehen, dass sie von ihren Mitspielern dank des Attraktivitätsstereotyps bevorzugt werden. Sie werden für die besseren Fußballer erachtet und können so ihre Fähigkeiten nicht nur häufiger unter Beweis stellen, sondern auch festigen und ausbauen - allein weil die anderen Spieler des Teams ihnen dazu die Gelegenheit geben. Selbst eklatante Fehlleistungen, wie beispielsweise das Verschießen eines eigentlich sicheren Tors, müssen diesen Vorteil nicht unbedingt beeinträchtigen. Sie lassen sich bagatellisieren (,Kann doch jedem einmal passieren“) oder wegrationalisieren (,Er konnte ja nichts dafür, der gegnerische Abwehrspieler hatte ihn vor dem Schuss unfair angerempelt und so aus dem Tritt gebracht"). Schließlich sollte ein attraktiver Fußballspieler auch von der Neigung zur Besserbehandlung schöner Menschen profitieren können. Denn im Zweifelsfall werden seine Mannschaftskameraden den Ball eher ihm zuspielen als dem weniger ansehnlichen Teamkollegen. Gegebenenfalls werden sie in kritischen Spielsituationen auch eher geneigt sein, ihm den Weg frei zu räumen. Und selbst die Spieler der gegnerischen Mannschaft lassen sich möglicherweise von seiner äußeren Anmutung beeindrucken und gehen ihn in Zweikämpfen weniger hart an, so dass er sich leichter durchsetzen kann. ${ }^{2}$

Wenn diese Einschätzungen stimmen und attraktive Fußballprofis auf Grund der günstigeren Umstände, die ihnen ihre äußere Anmutung verschafft, tendenziell mehr leisten können als ihre unattraktiven Teamkameraden, dann sollte eigentlich gelten, dass sie tendenziell auch mehr leisten müssten als diese - sofern keine intervenierenden Einflüsse zum Tragen kommen. Genau das ist aber der springende Punkt: Dass sie tendenziell mehr leisten können und leisten müssten heißt nicht unbedingt, dass sie auf dem Fußballplatz tendenziell auch tatsächlich mehr leisten werden als ihre unattraktiven Teamkameraden. Dank des Attraktivitätsstereotyps, des Glamour-Effekts und der Neigung zur Besserbehandlung schöner Menschen können sie nämlich auch für objektiv gesehen schlechtere Spielleistungen dieselbe oder höhere Anerkennung von Mannschaftskameraden, Trainern, Vereinsmanagern, Fans und Sportreportern ernten. Mit anderen Worten: Sie müssen weniger Leistung zeigen, um genauso erfolgreich zu sein wie ein weniger attraktiver Fußballprofi. Möglicherweise können sie sogar mit weniger Einsatz und Talent mehr Erfolg erringen. Im Umkehrschluss bedeutet das aber, dass unattraktive Fußballspieler mehr Leistung erbringen müssen, wenn sie dieses Handicap kompensieren wollen. Sie müssen die besseren Spieler sein, wenn sie im Vergleich zu ihren attraktiven Kollegen wenigstens als gleichwertige Spieler anerkannt sein wollen.

Aus denselben allgemein bekannten Wirkungsmechanismen der physischen Attraktivität lassen sich also mit Blick auf das sportliche Leistungsverhalten attraktiver und unattraktiver Individuen zwei einander diametral entgegengesetzte Hypothesen generieren: Zum einen lässt sich postulieren, dass attraktive Fußballspieler auf Grund der Vorteile, die ihnen ihr Aussehen möglicherweise verschafft, tendenziell höhere Leistungen als ihre weniger ansehnlichen Teamkameraden erbringen. Zum anderen lässt sich vermuten, dass genau diese Teamkameraden tendenziell höhere Leistungen erbringen, um die Wettbewerbsnachteile zu kompensieren, die sie gegenüber attraktiveren Fußballspielern haben. Welche dieser beiden Hypothesen Gültigkeit beanspruchen darf, lässt sich nur empirisch klären. Dabei muss allerdings berücksichtigt werden, dass es möglicherweise von den Kontextbedingungen abhängt, ob sich ein

2 Allerdings ist ebenfalls denkbar, dass attraktive Fußballspieler den Gegnern auf Grund des Attractiveness Stereotype als besonders gefährlich gelten und daher umso gewissenhafter abgedeckt werden. Dies würde dann allerdings einen Nachteil höherer Attraktivität bedeuten. 
positiver oder ein negativer Zusammenhang zwischen der physischen Attraktivität von Fußballprofis und ihren sportlichen Leistungen einstellt. Gemeint ist hier eine spezifische Variante des Attractiveness Frog Pond Effect (Rosar et al. 2008: 67 f). In welchem Umfang und mit welcher Wirkungsrichtung die physische Attraktivität eines Fußballprofis seine sportliche Leistung beeinflusst, dürfte nämlich durch die Attraktivität der übrigen Mannschaftsmitglieder moderiert werden. Je stärker die Attraktivitätsschere innerhalb eines Teams auseinander geht, desto mehr sollte das eben beschriebene Handicap unattraktiver Spieler ins Gewicht fallen und desto mehr sollten sie genötigt sein, diesen Nachteil durch verstärkte sportliche Leistungen auszugleichen. Je höher jedoch die durchschnittliche Attraktivität der Spieler eines Fußballteams ausfällt, desto geringer sollte der Anreiz für einzelne Spieler sein, sportliche Leistung durch physische Attraktivität zu kompensieren, und desto größer sollten die Chancen sein, dass sich die positiven Effekte der physischen Attraktivität auf dem Spielfeld entfalten und so zu einer verbesserten Spielleistung führen.

\section{Datenbasis: Alle Spitzenmannschaften, alle Spieltage, alle Spieler}

Unsere Untersuchung bezieht sich auf die 1. Fußballbundesliga der Spielsaison 2007/2008. Hier steht uns für jeden Spieltag für jeden eingesetzten Fußballspieler jeder Erstligamannschaft eine objektive Messung der sportlichen Leistung während des Fußballspiels zur Verfügung. Insgesamt verfügen wir über 8.420 Leistungsmessungen für 483 Fußballprofis von 18 Bundesligavereinen an 34 Spieltagen. ${ }^{3}$ Diese Leistungsmessungen wurden von der IMPIRE AG erstellt und geben für jeden Fußballprofi für jedes Fußballspiel, bei dem er eingesetzt wurde, die individuelle Leistung übersetzt in Bonus- und Maluspunkte wieder, die über den gesamten Einsatz aufsummiert werden und so die Gesamtleistung während des Spieleinsatzes reflektieren. Die IMPIRE AG ist Betreiberin der Fußball-Bundesliga-Datenbank. ${ }^{4}$ Bei jedem Bundesligaspiel setzt sie vier ausgebildete Spielbeobachter - so genannte Scouts - ein. Zwei von ihnen sitzen im Stadion und codieren live das Spielgeschehen. Dabei werden neben der taktischen Spielposition und der Einsatzdauer der einzelnen Fußballspieler bis zu 2.000 verschiedene Spielereignisse statistisch festgehalten. Einzelne Ereignisse wie beispielsweise gelbe und rote Karten werden nach Spielende noch einmal durch Rücksprache mit dem Schiedsrichterteam verifiziert. Darüber hinaus werden alle Bundesligaspiele auf Video aufgezeichnet und von zwei weiteren Experten noch einmal im Detail - gegebenenfalls mit Hilfe von Zeitlupen - analysiert und nachträglich codiert oder recodiert. So werden zum Beispiel bei Toren rund 20 Detailinformationen unterschieden. Anschließend werden alle Einzelleistungen und Einzelfehler eines Spielers anhand eines allgemeinen Wertungskataloges und eines speziellen, auf die Spielposition bezogenen Wertungskataloges in Punkte übersetzt. Dieses Standardpunktesystem erlaubt es, auch Leistungen von Fußballspielern zu vergleichen, die auf verschiedenen Positionen spielen $^{5}$. Beispielsweise bekommt ein Feldspieler, der ein Feldtor (kein Elfmeter) schießt, für diese Leistung +100 Punkte zugewiesen. Ein Torwart, der ein entsprechendes Gegentor erhält, bekommt hingegen -100 Punkte für diese Fehlleistung zugewiesen. Die meisten Codierungen lassen sich in dieser Weise als objektive Messungen verstehen, da genauestens definiert ist, für welche Aktionen wie viele Punkte vergeben werden. Beispielsweise gibt es einen be-

3 Am 28. Spieltag wurde die Partie zwischen dem 1. FC Nürnberg und dem VfL Wolfsburg abgebrochen und zu einem späteren Termin neu angesetzt. Die Spielereinsätze bei der abgebrochenen Begegnung konnten nicht berücksichtigt werden, da hier aus evidenten Gründen keine Leistungsmessung vorgenommen wurde. Bei den 483 Fußballspielern sind die acht Spieler doppelt gezählt, die während der laufenden Saison zwischen zwei Bundesligavereinen gewechselt haben und für beide Teams zum Einsatz kamen. Aus analysetechnischen Gründen war diese Vorgehensweise zwingend geboten.

4 Vgl. www.bundesliga-datenbank.de.

5 Neben 28 allgemeinen Wertungskriterien gibt es fünf bis acht positionsbezogene Wertungskriterien für Torhüter, Abwehr, Mittelfeld und Sturm. Weitere Details zum Wertungssystem können bei der Impire AG nachgefragt werden (vgl. zu den Kontaktdaten www.bundesliga-datenbank.de). 
stimmten Bonus für einen Führungstreffer und einen anderen für einen Ausgleichstreffer. Wenige Ereignisse haben auch eine als leicht subjektiv beschreibbare Dimension. Beispielsweise gibt es einen Punktabzug für denjenigen Spieler, der eine so genannte Großchance vergeben hat. Was eine Großchance ist, entscheiden die Spielbeobachter unmittelbar aus der Einschätzung des beobachteten Geschehens. Das Risiko subjektiver Verzerrungen ist dabei natürlich nicht von der Hand zu weisen, wird aber durch die intersubjektive Messung durch zwei Spielbeobachter pro Mannschaft und die nachträgliche Videokontrolle abgemildert. ${ }^{6}$ Weitgehend subjektive Qualitätszuschreibungen wie beispielsweise die Bewertung eines Passes als schön oder schlecht werden hingegen gar nicht vorgenommen. Die Gesamtpunktzahl, die ein Fußballspieler während eines Einsatzes erhalten kann, ist weder im negativen noch im positiven Bereich begrenzt. Sie findet ihre Grenzen lediglich dadurch, dass in einer Fußballbegegnung nicht unendlich viele Aktionen eines einzelnen Spielers stattfinden können.

Die Ermittlung der physischen Attraktivität der Fußballprofis gestaltete sich für uns etwas aufwändiger als die Bestimmung der sportlichen Leistung. Gemäß der in der Attraktivitätsforschung üblichen Vorgehensweise sollte die Attraktivitätsmessung anhand von Portraitphotographien erfolgen. Diese Photographien wurden von uns zunächst im Internet recherchiert, wobei wir nahezu durchgängig auf das Online-Angebot des Fußballmagazins kicker zurückgreifen konnten. Uns ist es dabei gelungen, für alle 483 Fußballprofis und einen weiteren, für die Untersuchungszwecke relevanten Spieler eine geeignete Portraitphotographie zu ermitteln. ${ }^{7}$ Nahezu alle Photographien wurden im Sommer 2007 aufgenommen und spiegeln daher die äußere Anmutung der Fußballspieler in etwa zum selben Zeitpunkt wieder. Im Rahmen der Photorecherche konnten wir auch für jeden der 483 Fußballprofis weitere relevante Daten sammeln, auf deren Grundlage später Variablen erstellt werden konnten, die als Kontrollmerkmale Eingang in die Untersuchung finden sollten. Auch dabei konnten wir uns nahezu durchgängig auf das Online-Angebot des kicker stützen.

Die Portraitphotographien der insgesamt 484 Fußballspieler wurden von uns in einem nächsten Schritt auf eine einheitliche Höhe formatiert und in einen Online-Fragebogen eingearbeitet. Jede Photographie wurde einer separaten Fragebogenseite zugewiesen und um eine siebenstufige Skala zur Attraktivitätsbeurteilung ergänzt. Die Endpole dieser Skala wurden mit unattraktiv (im Datensatz später mit dem Wert 0 codiert) und attraktiv (im Datensatz später mit dem Wert 6 codiert) gelabelt. Keine der Seiten mit den Photographien enthielt Informationen zu der gezeigten Person. Da dennoch davon auszugehen war, dass der eine oder andere Fußballer im Rahmen der Erhebung erkannt werden könnte, und nicht ausgeschlossen werden konnte, dass dies Auswirkungen auf die Attraktivitätsbeurteilung hat, wurde auf jeder Fragebogenseite auch nach der Bekanntheit und dem Namen der gezeigten Person gefragt. Um Ermüdungseffekte zu vermeiden und Reihenfolgeeffekte ausgleichen zu können, wurden zudem Zufallsfilter programmiert. Sie bewirkten zum einen, dass jeder Erhebungsteilnehmer später nur die Fußballspieler einer Mannschaft sah, und zum anderen sorgten sie dafür, dass

6 Man kann die Daten der IMPIRE AG auch als eine von allen Marktteilnehmern anerkannte Leistungswährung für Fußballer beschreiben. Das lässt sich daran erkennen, dass Marktteilnehmer mit unterschiedlichsten Interessen wie Fernsehsender, Zeitungen, Zeitschriften oder Fußballvereine die Daten beziehen und sie für aktuelle Leistungsbewertungen (durch Kommentatoren, bei Notengebungen, für die Trainingssteuerung) oder längerfristige Dokumentationen nutzen (z.B. Bundesliga-Jahrbuch der ARD-Sportschau).

7 Bei dem zusätzlichen Spieler handelt es sich um einen Fußballprofi, der in der Spielzeit 2007/2008 beim VfL Wolfsburg unter Vertrag stand und der nur bei der abgebrochenen Partie mit dem 1. FC Nürnberg zum Einsatz kam. Da er dadurch zwar nicht zur Grundgesamtheit der Untersuchung gehört, gleichzeitig aber dennoch dem Mannschaftskader des VfL Wolfsburg zuzurechnen ist, war sein Attraktivitätsscore wichtig, um für den VfL Wolfsburg den Frog Pond-Effekt adäquat modellieren zu können. 
die Reihenfolge, in der diese Spieler präsentiert wurden, für jeden Erhebungsteilnehmer neu festgelegt wurde.

Die eigentliche Messung der Attraktivität erfolgte nach der Truth of Consensus Method (Patzer 1985: 17). Hierbei beurteilt eine Gruppe von so genannten Ratern unabhängig voneinander die Attraktivität der ihnen gezeigten Personen. Durch Verrechnung der Einzelbewertungen zu einem Mittelwert ergibt sich dann der Attraktivitätsscore der beurteilten Person. Grundlage dieses Verfahrens ist der bereits angesprochene Attraktivitätskonsens, also die Tatsache, dass die Attraktivität ein objektives Merkmal der betrachteten Person ist, das unterschiedliche Betrachter sehr ähnlich einschätzen (vgl. Grammer et al. 2003; Henss 1987; 1992; Iliffe 1960). Auf Grund der hohen intersubjektiven Übereinstimmung zwischen verschiedenen Betrachtern kann schon mit einer vergleichsweise kleinen Gruppe von Ratern eine zufriedenstellend reliable und valide Attraktivitätsmessung erfolgen. In der einschlägigen Literatur wird es gemeinhin als hinreichend erachtet, wenn die Attraktivitätsmessung auf den Urteilen von rund zwei Dutzend Ratern aufbaut. Bereits dann kann davon ausgegangen werden, dass der Attraktivitätsscore so stabil ist, dass sich auch mit einer größeren Anzahl von Ratern kaum ein anderes Ergebnis einstellen würde (Henss 1992: 308).

Als Rater für die Attraktivitätsbestimmung der Fußballprofis dienten uns 365 männliche Teilnehmer des Online-Access-Panels Sozioland der Respondi $A G$ im Alter zwischen 17 und 64 Jahren. ${ }^{8}$ Die Rater konnten den Fragebogen von ihrem heimischen Computer aus bearbeiten und wurden uns von der Respondi $A G$ kostenlos zur Verfügung gestellt. ${ }^{9}$ Jede Photographie wurde im Durchschnitt von 20 Ratern auf der von unattraktiv (0) bis attraktiv (6) reichenden siebenstufigen Skala bewertet. ${ }^{10}$ Die zu erwartende deutliche Übereinstimmung in den Attraktivitätsurteilen der Rater ließ sich durch eine Reliabilitätsanalyse bestätigen. Gemäß der in der Attraktivitätsforschung üblichen Vorgehensweise wurden dabei die Rater als Variablen und die präsentierten Photographien als Fälle betrachtet. Für Cronbach's alpha ergab sich dabei im Durchschnitt ein Wert von $0,81 .{ }^{11}$ Die Sorge, dass die Attraktivitätsmessung möglicherweise durch das Wiedererkennen einzelner Fußballspieler verzerrt sein könnte, erwies sich hingegen im Nachhinein als unbegründet. Lediglich bei 42 von insgesamt 9.888 Messungen (0,4 Prozent) wurde die gezeigte Person erkannt, so dass die Möglichkeit nennenswerter Verzerrungen auf Grund von Bekanntheit vernachlässigt werden kann. Aus den einzelnen Attraktivitätsurteilen der Rater wurde daher - gemäß der Truth of Consensus Method - für jeden Fußballer durch Mittelwertbildung ein Attraktivitätsscore berechnet. Im Durchschnitt ergab sich dabei ein Attraktivitätsscore von 2,26. Der schlechteste Wert beträgt 0,53, der beste 3,95 und die Standardabweichung liegt bei 0,63. Um einen plastischen Eindruck von den Merkmalen zu geben, die bei Männern eine positive Attraktivitätsbeurteilung begünstigen, ist in Abbildung 1 die Zusammensetzung der zehn attraktivsten Fußballspieler dargestellt. Die physiologischen Merkmale, die diese zehn Gesichter aufweisen, spiegeln gut die bekannten Befunde der Attraktivitätsforschung wieder (vgl. zusammenfassend Braun et al. 2003: $42 \mathrm{f}$.): wenig Fettansatz, eher schmale und längliche Gesichtsform, relativ volle Lippen, höhere Wangenknochen, keine Geheimratsecken und kein Ansatz zur Glatze.

8 Hier und im Folgenden sind nur die Rater berücksichtigt, die alle ihnen gezeigten Photographien bewertet haben.

9 An dieser Stelle möchten wir uns herzlich bei der Respondi $A G$ bedanken. Die Untersuchung wäre ohne die großzügige Unterstützung durch die Respondi $A G$ in der hier vorliegenden Form überhaupt nicht möglich gewesen.

10 Das arithmetische Mittel der Zahl der Rater beträgt 20,43 und der Median 20,00. Das Minimum ist 14, das Maximum 26. Die Standardabweichung liegt bei 3,57.

11 Das Minimum liegt bei 0,72, das Maximum bei 0,87, der Median liegt bei 0,83 und die Standardabweichung beträgt 0,04 . 


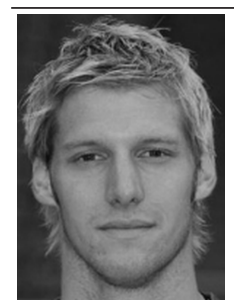

3,95

geb. 1980

Markus Daun

MSV Duisburg

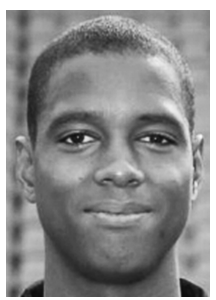

3,53

geb. 1986

Ricardo Faty

Bayer 04 Leverkusen

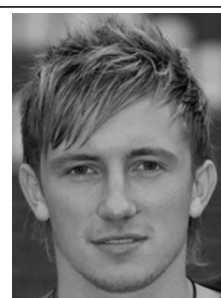

3,65

geb. 1985

Adam Bodzek

MSV Duisburg

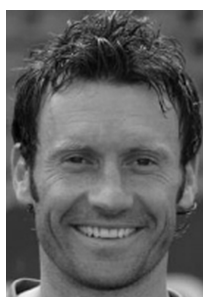

3,53

geb. 1973

Thomas Zdebel

VfL Bochum

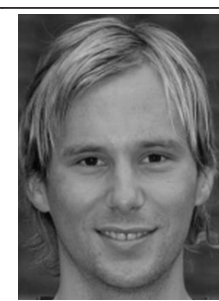

3,65

geb. 1986

Markus Neumayr

MSV Duisburg

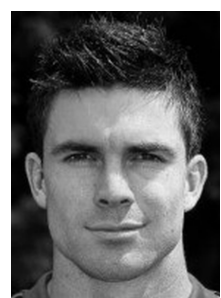

3,50

geb. 1981

Michael Beauchamp

1. FC Nürnberg

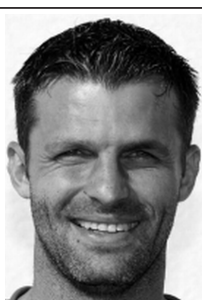

3,60

geb. 1975

Mieciel Marcin

VfL Bochum

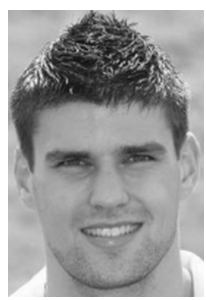

3,50

geb. 1984

Florian Dick

Karlsruher SC

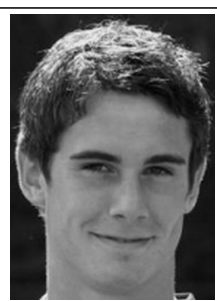

3,57

geb. 1987

Fin Bartels

Hansa Rostock

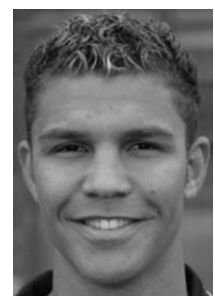

3,50

geb. 1985

Daniel Gordon

BVB Dortmund

Anmerkungen: Angaben unter den Photographien sind der Attraktivitätsscore, das Geburtsjahr, der Name und die Mannschaft des Fußballspielers.

Bevor wir uns nun den Befunden der empirischen Analysen zuwenden, sind noch zwei abschließende Anmerkungen zur Datenbasis erforderlich. Erstens muss berücksichtigt werden, dass unsere Daten eine hierarchische Struktur aufweisen, bei der die fußballerischen Leistungen der Spieler die erste Datenebene bilden und die Attraktivitätsscores der Spieler auf der zweiten Datenebene angesiedelt sind. Wenn im Fortgang der empirischen Analysen die Attraktivitätskennwerte der Mannschaften einbezogen werden, dann ergibt sich eine dritte Datenebene. Das angemessene Verfahren zur Analyse derart hierarchisch strukturierter Daten ist die Mehrebenenanalyse (vgl. zum Verfahren Bryk / Raudenbush 1992; Hox 2002; Snijders / Bosker 1999). Entsprechend kam sie bei allen nachfolgend berichteten statistischen Auswertungen zur Anwendung. Zweitens ist zu beachten, dass wir für alle Untersuchungseinheiten der ersten, der zweiten und der dritten Ebene alle relevanten Daten erfassen konnten. Alle unsere nachfolgend berichteten empirischen Analysen beziehen sich also auf eine Vollerhebung, deswegen werden im Folgenden keine Signifikanzen berichtet.

\section{Empirische Analysen: Physische Attraktivität, physische Konstitution, Spielsituation, Franck Ribéry und der Frog Pond-Effekt}

Die abhängige Variable aller berechneten Analysemodelle bilden die Leistungspunktsalden, die sich aus den Daten der IMPIRE AG für jeden Spieler für jeden Einsatz bei einem Spiel der 1. Bundesliga in der Saison 2007/2008 ergeben. In einem ersten Modellierungsschritt wurden zunächst keine Prädiktoren auf der ersten Datenebene eingeführt, und das Interzept der Leistungspunktesalden wurde alleine in Abhängigkeit von der physischen Attraktivität der Spieler 
geschätzt (Modell 1 in Tabelle 1). Dabei zeigte sich, dass die physische Attraktivität einen negativen und substantiell bedeutsamen Einfluss auf die Spielleistung hat. Mit jedem Skalenpunkt, um den der Attraktivitätsscore steigt, sinkt die Leistung im Durchschnitt um 10,77 Punkte. Berücksichtigt man, dass die Spannweite bei den Attraktivitätsscores der Spieler 3,42 Skalenpunkte ${ }^{12}$ beträgt, so ergibt sich ein Gesamteffekt von knapp 37 Punkten bei der durchschnittlichen Leistung pro Spiel.

Unattraktive Fußballer scheinen also nach den Befunden des Modells 1 in Tabelle 1 die leistungsstärkeren Fußballer zu sein. Allerdings berücksichtigt dieses Modell noch keine Kontrollvariablen, die ebenfalls die Leistung beeinflussen können und - zumindest teilweise - mit der physischen Attraktivität konfundiert sein könnten. Denkbar sind hier sowohl Merkmale der physischen Konstitution eines Spielers als auch Einflüsse, die sich aus der Spielsituation ergeben. In einem weiteren Modellierungsschritt haben wir daher entsprechende Kontrollmerkmale in das Analysemodell eingeführt (vgl. Modell 2 in Tabelle 1). Die physische Konstitution haben wir dabei über den Body Mass Index der Spieler ${ }^{13}$, ihre Körpergröße ${ }^{14}$ und ihr Alter ${ }^{15}$ erfasst. Sie wurden auf der zweiten Ebene in das Modell eingeführt. Die Implikationen der Spielsituation haben wir über die Einsatzdauer während eines Spiels ${ }^{16}$, die Spielposition $^{17}$, den Spieltag ${ }^{18}$, den Spielort ${ }^{19}$, den Tabellenplatz der Mannschaft vor dem Spieltag ${ }^{20}$, den

12 Vgl. dazu noch einmal den vorletzten Absatz in Abschnitt 2.

13 Da wir es in unserer Untersuchung mit durchtrainierten Spitzensportlern zu tun haben, gehen wir davon aus, dass ein höherer Body Mass Index ein Mehr an Muskelmasse indiziert und nicht, wie beim Durchschnittsmann von der Straße üblich, ein Mehr an Fettmasse. In sportlichen Zweikämpfen oder Laufduellen auf dem Spielfeld sollte ein Mehr an Muskeln aber einen Vorteil darstellen, so dass davon auszugehen ist, dass sich mit zunehmendem Body Mass Index die fußballerische Leistung verbessert. Der Form halber weisen wir darauf hin, dass wir den Body Mass Index in einer anderen Abhandlung als Indikator der Körperattraktivität interpretieren und im Rahmen der dort berechneten Kausalmodelle als Prädiktor des Marktwertes von Fußballprofis nutzen. Dies geschieht aber bei gleichzeitiger Berücksichtigung der sportlichen Leistungen, so dass der Aspekt der spielerischen Überlegenheit statistisch kontrolliert wird (vgl. Rosar et al. 2010).

14 Bei der Körpergröße gehen wir davon aus, dass große Fußballer auf dem Spielfeld, beispielsweise bei Kopfballduellen, im Vorteil sind. Entsprechend erwarten wir einen positiven Einfluss auf die sportliche Leistung.

15 Mit zunehmendem Alter sinkt die körperliche Leistungsfähigkeit. Wir gehen daher davon aus, dass die sportliche Leistung älterer Fußballspieler geringer ist als die jüngerer Spieler.

16 Die Leistungspunktesalden der IMPIRE AG sind nicht für die tatsächliche Dauer des Spieleinsatzes standardisiert. Insofern muss mit zunehmender Einsatzdauer die Zahl der Leistungspunkte tendenziell ansteigen. Hinzu kommt, dass sich mit zunehmender Einsatzdauer ein besseres Gefühl für die Dynamik des Spiels entwickeln sollte, so dass es für den Fußballer leichter wird, Schwächen des Gegners zu erkennen und auszunutzen.

17 Wir gehen davon aus, dass Feldspieler größere Spielanteile als Torhüter haben. Entsprechend sollte ihre Chance, ihre Leistungsfähigkeit in tatsächliche Leistungen umzusetzen, größer sein. Die Spielposition ging daher in Dummycodierung in das Analysemodell ein, wobei die Position des Torhüters die Referenzkategorie bildet. Um gegebenenfalls Unterschiede zwischen verschiedenen Feldpositionen nicht zu verwischen, haben wir bei den Feldpositionen zusätzlich nach Abwehr, Mittelfeld und Sturm differenziert.

18 Eine Bundesligasaison ist lang und Kräfte zehrend. Entsprechend sollte die fußballerische Leistung mit dem Fortschreiten der Spieltage tendenziell nachlassen.

19 Es ist eine fußballerische Binsenweisheit, dass die Heimmannschaft im Vorteil ist. Entsprechend sollten sich die fußballerischen Leistungen der Spieler verbessern, wenn die Partie auf eigenem Rasen ausgetragen wird (vgl. Skarupke 2000).

20 Wir nehmen an, dass der Tabellenplatz, von dem aus eine Mannschaft in eine aktuelle Spielbegegnung geht, das Selbstbewusstsein der Mannschaftsmitglieder beeinflusst und dass dies Konsequenzen für die Leistung jedes einzelnen Spielers hat. Je besser dabei die Positionierung in der Tabelle, desto höher das Selbstbewusstsein der Spieler und desto besser die von ihnen gezeigte Leistung. Da vor dem ersten Spieltag noch keine Tabellenplatzierungen bestehen, haben wir stattdessen auf die Tabellenplatzierung der Mannschaften am Ende der Bundesligasaison 2006/2007 zurückgegriffen. Den 
Abstand zum Tabellenplatz der gegnerischen Mannschaft vor dem Spieltag ${ }^{21}$ sowie einem Interaktionsterm zwischen dem Spieltag und dem Abstand zum Tabellenplatz der gegnerischen Mannschaft vor dem Spieltag 22 operationalisiert. Da diese Merkmale zwischen den Spieltagen variieren können bzw. zwangsläufig variieren müssen, wurden sie auf der ersten Ebene in das Modell eingeführt. Die Berücksichtigung der physischen Konstitution und der Spielsituation hat jedoch nur marginale Auswirkungen auf die Stärke des Effekts, der von der physischen Attraktivität der Fußballspieler auf ihr Leistungsverhalten ausgeht. Nach wie vor erweisen sich unattraktive Fußballprofis als die leistungsstärkeren Spieler. ${ }^{23}$

Wir wollen jedoch nicht verschweigen, dass dieser Befund maßgeblich durch einen einzelnen Fußballspieler beeinflusst sein könnte. Dieser Spieler ist Franck Ribéry vom FC Bayern München. Franck Ribéry ist einer der leistungsstärksten Fußballer der Spielsaison 2007/2008, gleichzeitig ist er aber auch einer der unattraktivsten Fußballer. ${ }^{24}$ Theoretisch lässt sich nicht ausschließen, dass diese Koinzidenz die bisherigen Modellschätzungen zum Einfluss der physischen Attraktivität deutlich beeinflusst hat. Empirisch ist dies jedoch nicht der Fall. Das geht aus Modell 3 in Tabelle 1 hervor. Das Modell entspricht weitgehend dem Modell 2 in Tabelle 1. Der einzige Unterschied besteht darin, dass auf der zweiten Ebene eine Dummyvariable für die Person Franck Ribérys einbezogen wurde. Der Effekt der physischen Attraktivität wird dadurch zwar etwas schwächer, die negative Beeinflussung der fußballerischen Leistung bleibt als Tendenz jedoch bestehen.

Bundesligaaufsteigern wurden dabei - gemäß ihrer Rangfolge in der 2. Bundesliga - die Tabellenplätze 16,17 und 18 zugewiesen.

21 Für jeden einzelnen Fußballspieler dürfte es umso schwerer sein, durch sportliche Leistungen zu glänzen, je besser der Gegner in einer Partie ist. Um diesen Effekt abbilden zu können, haben wir eine Proxyvariable modelliert, die, bezogen auf den Tabellenplatz vor dem Anpfiff, das Differenzial zwischen der Mannschaft eines Spielers und der gegnerischen Mannschaft in dieser Begegnung wiedergibt. Dieses Differenzial kann Werte zwischen -17 und +17 annehmen. Positive Werte indizieren, dass die Mannschaft dieses Fußballspielers überlegen ist. Negative Werte indizieren, dass die gegnerische Mannschaft überlegen ist. Für die Begegnungen des ersten Spieltages wurde wie bei der Variable zum Tabellenplatz der Mannschaft verfahren (vgl. nochmals Fußnote 20).

22 Es muss davon ausgegangen werden, dass die Tabellenplatzierung die spielerische Stärke der Mannschaften zu Saisonbeginn wesentlich schlechter widerspiegelt als zum Ende der Saison, da zu Anfang kleine Zufallserfolge einen deutlichen Sprung in der Tabelle bewirken können. Um diese Ungenauigkeit ausgleichen zu können, wurde dieser Interaktionsterm zusätzlich eingeführt.

23 Da die Wirkung der physischen Attraktivität im Fokus unserer Untersuchung steht, gehen wir hier nicht auf die Effekte der Kontrollvariablen ein. Nur soviel sei dazu angemerkt: Alle Effekte der Kontrollvariablen weisen ein erwartungskonformes Vorzeichen auf, bis auf einen. Dieser kontraintuitive Effekt geht vom Tabellenplatz der Mannschaft aus und weist ein positives Vorzeichen statt eines negativen auf. Offensichtlich ist es also nicht so, dass eine schlechte Tabellenplatzierung das Selbstbewusstsein der Teammitglieder beeinträchtigt. Vielmehr scheint es so zu sein, dass dies anspornend wirkt, mehr Leistung zu erbringen, um so die Tabellenplatzierung der eigenen Mannschaft zu verbessern.

24 Mit einer durchschnittlichen Leistung von +280 Punkten pro Spieleinsatz ist er über die gesamte Saison betrachtet der zweitbeste Fußballspieler. Gleichzeitig ist er gemäß den Befunden unserer Attraktivitätsmessung jedoch auch der viertunattraktivste Spieler. 
Tabelle 1: Zwei-Ebenen-Modelle der Determinanten der sportlichen Leistung der Fußballspieler der 1. Bundesliga-Spielsaison 2007/2008

\begin{tabular}{|c|c|c|c|}
\hline $\begin{array}{l}\text { Level } 1 \text { (Spieleinsätze) } \\
\text { Level } 2 \text { (Spieler) }\end{array}$ & Modell 1 & Modell 2 & Modell 3 \\
\hline \multicolumn{4}{|l|}{ Fixe Effekte } \\
\hline \multicolumn{4}{|l|}{ Interzept 1 (Spieleinsätze) } \\
\hline Interzept 2 (Spieler) & 114,21 & $-314,69$ & $-331,24$ \\
\hline Physische Attraktivität des Fußballers & $-10,77$ & $-8,74$ & $-7,25$ \\
\hline Body Mass Index des Fußballers & & 5,42 & 4,84 \\
\hline Körpergröße des Fußballers in cm & & 82 & ,94 \\
\hline Alter des Fußballers in Jahren & & $-1,45$ & $-1,31$ \\
\hline Personendummy: Franck Ribéry & & & 138,41 \\
\hline Einsatzdauer des Fußballers in Minuten & & 1,56 & 1,56 \\
\hline \multicolumn{4}{|l|}{ Position des Fußballers } \\
\hline Abwehr & & 18,07 & 18,31 \\
\hline Mittelfeld & & 68,61 & 68,51 \\
\hline Sturm & & 89,51 & 89,72 \\
\hline Spieltag & &,- 71 &,- 71 \\
\hline Heimspiel & & 43,51 & 43,49 \\
\hline Tabellenplatz der Mannschaft vor dem Spiel & & ,76 & ,73 \\
\hline Tabellenplatz-Differenzial Gegenmannschaft - Mannschaft & & 1,45 & 1,44 \\
\hline Spieltag * Tabellenplatz-Differenzial & & ,06 & ,06 \\
\hline \multicolumn{4}{|l|}{ Zufallseffekte } \\
\hline Varianz des Interzept 1 (Spieleinsätze) & $2.186,22$ & 947,62 & 893,39 \\
\hline Devianz & 105.362 & 104.164 & 104.151 \\
\hline Anzahl der Parameter & 4 & 16 & 17 \\
\hline Level 1-N (Spieleinsätze) & 8.420 & 8.420 & 8.420 \\
\hline Level 2-N (Spieler) & 483 & 483 & 483 \\
\hline
\end{tabular}

Bleibt noch zu klären, ob die Wirkung der physischen Attraktivität in der erwarteten Weise durch einen Frog Pond-Effekt moderiert wird. Zu diesem Zweck haben wir zwei weitere Mehrebenenmodelle geschätzt, die auf einer dritten Datenebene die physische Gesamtanmutung der Mannschaften einbeziehen. Die Gesamtanmutung der Mannschaften wurde dabei zum einen über die durchschnittliche Attraktivität der Mannschaftsmitglieder operationalisiert. Zum anderen wurde sie über die Spannweite zwischen den Attraktivitätsscores des unattraktivsten Mannschaftsmitglieds und des attraktivsten Mannschaftsmitglieds erfasst. Sofern sich ein erwartungskonformer Frog Pond-Effekt einstellt, sollte die durchschnittliche Mannschaftsattraktivität den Effekt, der von der individuellen Attraktivität der Spieler ausgeht, positiv beeinflussen, und die Spannweite in den Attraktivitätswerten eines Mannschaftskaders sollten ihn negativ beeinflussen. Die in Modell 1 in Tabelle 2 ausgewiesenen Befunde bestätigen diese Erwartungen. Mit jedem Skalenpunkt, um den sich die Durchschnittsattraktivität einer Mannschaft erhöht, verstärkt sich der Effekt der Attraktivität auf Spielerebenen im Mittel um 2,88 Leistungspunkte. Und mit jedem Skalenpunkt, um den sich die Attraktivitätsspreizung innerhalb einer Mannschaft erhöht, reduziert sich der Attraktivitätseffekt auf der Ebene der Spieler im Durchschnitt um 12,03 Leistungspunkte. Beachtenswert ist aber auch, dass der Basiseffekt 
der Spielerattraktivität in diesem Modell positiv ist. In der Grundtendenz gilt also, dass physische Attraktivität leistungsfördernd ist. Setzt man für die einzelnen Mannschaften die konkreten Kennwerte für die mittlere Attraktivität und die Attraktivitätsspreizung ein, so zeigt sich, dass der Effekt der Spielerattraktivität auf die Leistung im Durchschnitt zwischen -7,53 (MSV Duisburg) und +5,61 (VfL Wolfsburg) variiert. Für 13 der 18 Mannschaften ergibt sich dabei allerdings ein negativer Einfluss der Spielerattraktivität.

Auch die Einbeziehung des Ribéry-Effekts ändert diese Befunde nicht wesentlich (vgl. Modell 2 in Tabelle 2). Der Basiseffekt der Spielerattraktivität ist etwas stärker und die moderierenden Einflüsse der Durchschnittsattraktivität und der Attraktivitätsspreizung auf Mannschaftsebene sind etwas schwächer. Die negativen Effekte, die in den Modellen der Tabelle 1 für die physische Attraktivität ausgewiesen wurden, dürften damit vor allem darauf zurückzuführen sein, dass die Kader der 18 untersuchten Bundesligamannschaften im Durchschnitt eher unattraktiv sind, gleichzeitig aber eine starke Spreizung in den Attraktivitätsscores der Mannschaftsmitglieder aufweisen. Es sind also die faktischen Gegebenheiten und nicht grundsätzliche Mechanismen, die dazu führen, dass sich die physische Attraktivität bei der Population unserer Untersuchung tendenziell negativ auf die Spielleistung auswirkt.

Tabelle 2: Drei-Ebenen-Modelle der Determinanten der sportlichen Leistung der Fußballspieler der 1. Bundesliga-Spielsaison 2007/2008

\begin{tabular}{lrr}
\hline Level 1 (Spieleinsätze) & Modell 1 & Modell 2 \\
$\quad$ Level 2 (Spieler) & & \\
$\quad$ Level 3 (Mannschaften) & & \\
\hline Fixe Effekte & & \\
Interzept 1 (Spieleinsätze) & $-300,58$ & $-319,31$ \\
$\quad$ Interzept 2 (Spieler) & & \\
$\quad$ Interzept 3 (Mannschaften) & 19,50 & 21,63 \\
$\quad$ Physische Attraktivität des Fußballers & 2,88 & 1,73 \\
$\quad$ Interzept 3 (Basiseffekt) & $-12,03$ & $-11,36$ \\
$\quad$ Durchschnittliche physische Attraktivität der Mannschaft & 3,12 & 2,86 \\
$\quad$ Range der physischen Attraktivität der Mannschaft &, 59 &, 70 \\
$\quad$ Body Mass Index des Fußballers &,- 40 &,- 32 \\
$\quad$ Körpergröße des Fußballers in cm & & 90,20 \\
$\quad$ Alter des Fußballers in Jahren & 1,58 & 1,57 \\
$\quad$ Personendummy: Franck Ribéry & & \\
Einsatzdauer des Fußballers in Minuten & 15,43 & 16,07 \\
Position des Fußballers & 64,86 & 65,42 \\
$\quad$ Abwehr & 87,22 & 87,69 \\
$\quad$ Mittelfeld &,- 56 &,- 56 \\
$\quad$ Sturm & 42,49 & 42,46 \\
Spieltag & 4,85 & 4,84 \\
Heimspiel & 1,68 & 1,68 \\
Tabellenplatz der Mannschaft vor dem Spiel &, 04 &, 04 \\
Tabellenplatz-Differenzial Gegenmannschaft - Mannschaft & & \\
Spieltag * Tabellenplatz-Differenzial & 587,15 & 569,60 \\
Zufallseffekte & $2.456,18$ & $1.893,62$ \\
$\quad$ Varianz des Interzept 1 (Spieleinsätze) & 37,62 & 13,48 \\
Varianz des Interzept 2 (Spieler) & 104.032 & 104.026 \\
$\quad$ Varianz des Basiseffektes der physischen Attraktivität & 21 & 22 \\
\hline Devianz & 8.420 & 8.420 \\
Anzahl der Parameter & 483 & 483 \\
Level 1-N (Spieleinsätze) & 18 & 18 \\
Level 2-N (Spieler) & & \\
Level 3-N (Mannschaften) & & \\
\hline & & \\
\hline
\end{tabular}




\section{Fazit: Physische Attraktivität wirkt - und der Frog Pond moderiert}

Am Beispiel des Profifußballs der 1. Bundesliga in der Spielsaison 2007/2008 konnten wir empirisch klären, ob sich die physische Attraktivität positiv oder negativ auf individuelles Leistungsverhalten auswirkt. Vor dem Hintergrund der bisherigen Erkenntnisse der Attraktivitätsforschung erschienen beide Wirkungsrichtungen zunächst einmal gleichermaßen plausibel. Durch die empirischen Analysen zeigte sich jedoch, dass zwischen der physischen Attraktivität eines Fußballprofis und seiner sportlichen Leistung tendenziell ein negativer Zusammenhang besteht. Wir konnten aber auch zeigen, dass dieser Zusammenhang durch Kontextfaktoren moderiert wird und dass sich unter bestimmten Voraussetzungen - hohe Attraktivität und Homogenität der Mannschaft - der Effekt der physischen Attraktivität umkehrt.

Obwohl dieser Befund bereits für sich genommen bedeutsam ist, erschließt sich seine wirkliche Tragweite erst, wenn die Implikationen berücksichtigt werden, die sich aus ihm ergeben könnten. Sofern nämlich Fußballtrainer und Vereinsmanager realisieren, dass die Zusammensetzung, die eine Mannschaft hinsichtlich der physischen Attraktivität aufweist, das Leistungsverhalten der einzelnen Fußballspieler beeinflusst, könnten sie versucht sein, ihre Mannschaften (auch) nach Attraktivitätskriterien zusammenzustellen. Da in der Grundtendenz die positiven Effekte der physischen Attraktivität überwiegen dürften und gut aussehende Fußballer als Publikumsmagneten und unter Merchandising-Gesichtspunkten für die Vereine ohnehin vorteilhafter sein sollten, könnte dies zu einer weiteren Diskriminierung unattraktiver Fußballer führen. Sie würden dann nicht nur auf Grund der bereits bekannten Mechanismen benachteiligt, sondern auch, weil sich die Verantwortlichen in den Vereinen von ihrer Diskriminierung eine Verbesserung des Teamgefüges versprechen.

Natürlich kann man an unsere Untersuchung die Frage richten: So what? - Obwohl der bundesdeutsche Profifußball ein großes Publikum hat und unter ökonomischen Gesichtspunkten durchaus eine gewisse volkswirtschaftliche Rolle spielt, ist seine soziologische Bedeutung doch eher marginal. Dem würden wir selbstverständlich zustimmen. Aus unserer Sicht spricht jedoch nichts dagegen, die von uns ermittelten Befunde auf andere, soziologisch relevantere Handlungsfelder zu übertragen. Im Gegenteil: in Handlungsfeldern, in denen individuelle Leistung weniger eindeutig definiert und objektiv messbar ist, mag es sogar sein, dass die von uns am Beispiel des Profifußballs aufgezeigten Mechanismen noch bedeutsamer sind.

So ist es beispielsweise denkbar, dass analoge Effekte im Bildungssystem bestehen; dass weniger attraktive Schulkinder - bei entsprechender Verteilung der Attraktivität auf Klassenebene - mehr leisten bzw. leisten müssen, um die Nachteile, die sie gegebenenfalls gegenüber attraktiveren Klassenkameraden bei Entwicklungsprognosen, bei Empfehlungen für weiterführende Schulen oder bei der Notenvergabe durch das Lehrpersonal haben, zumindest teilweise zu kompensieren. Ebenso erscheint es naheliegend, dass sich dieses Muster im Rahmen der Berufsausbildung oder Hochschulbildung repliziert. Und nicht zuletzt mit Blick auf den Erwerbssektor lässt sich mutmaßen, dass Einstellungs- und Karrierechancen, der Erfolg bei Gehaltsverhandlungen oder das Risiko eines Arbeitsplatzverlustes in derselben Weise von der physischen Attraktivität beeinflusst werden.

Die einzige Einschränkung, die wir mit Blick auf die Übertragbarkeit unserer Befunde auf andere, soziologisch relevantere Handlungsfelder vornehmen würden, ergibt sich aus dem so genannten Beauty Is Beastly-Effect (vgl. Heilman / Saruwatari 1979; sowie die weiterführenden Arbeiten von Gillen 1981; Friedman / Zebrowitz 1992; Rosar / Klein 2009; Sczesny 2003; vgl. aber auch die kritischen Anmerkungen bei Podratz / Dipboye 2002). Mit diesem Begriff wird in der Attraktivitätsforschung das Faktum umschrieben, dass die Wirkungsrichtung der Attraktivität vom Geschlecht des Akteurs und von der geschlechtsspezifischen Konnotation des Handlungskontextes moderiert wird. So gilt etwa für Frauen, die in männlich dominierten 
Handlungskontexten - wie beispielsweise der akademischen Forschung - agieren, dass sich ihre physische Attraktivität negativ auf die Bewertung ihrer Leistungen auswirken kann. In diesem Fall müssten attraktive Frauen mehr leisten, um dieselbe Anerkennung wie ihre weniger attraktiven Geschlechtsgenossinnen und ihre männlichen Kollegen zu erhalten. In Verbindung mit unseren Befunden zum Attractiveness Frog Pond Effect ergibt sich durch den Beauty Is Beastly-Effect also gegebenenfalls eine komplexe Interaktion, die es zu beachten gilt, wenn im Zusammenhang mit der physischen Attraktivität Vorhersagen über das konkrete Leistungsverhalten in spezifischen sozialen Kontexten gemacht werden sollen. Da sich unsere Untersuchung nur auf Männer in einem klar männlich konnotierten Handlungskontext bezog, war es leider nicht möglich, diese Wechselwirkung in die Analyse einzubeziehen. In diesem Punkt halten wir es daher mit Sepp Herberger: Nach dem Spiel ist vor dem Spiel.

\section{Literatur}

Anderson, Cameron / John, Oliver P. / Keltner, Dacher / Kring, Ann M. (2001): Who attains social status? Effects of personality traits and physical attractiveness in social groups, in: Journal of Personality and Social Psychology 81, S. 116-132.

Bassili, John N. (1981): The attractiveness stereotype: Goodness or glamour?, in: Basic and Applied Social Psychology 2, S. 235-252.

Baugh, S. Gayle / Parry, Linda E. (1991): The relationship between physical attractiveness and grade point average among college women, in: Journal of Social Behavior and Personality 6, S. 219-228.

Berger, Roger / Hammer, Rupert (2007): Die doppelte Kontingenz von Elfmeterschüssen. Eine empirische Analyse, in: Soziale Welt 58, S. 397-418.

Benoy, Joseph W. (1982): The Credibility of Physically Attractive Communicators: A Review, in: Journal of Advertising 11, S. 15-24.

Benson, Peter L. / Karabenic, Stuart A. / Lerner, Richard M. (1976): Pretty Pleases: The effects of physical attractiveness on race, sex and receiving help, in: Journal of Experimental Social Psychology 12, S. 409-415.

Biddle, Jeff E. / Hamermesh, Daniel S. (1998): Beauty, Productivity, and Discrimination: Lawyers' Looks and Lucre, in: Journal of Labor Economics 16, S. 172-201.

Braun, Christoph / Gründl, Martin / Marberger, Claus / Scherber, Christoph (2003): Beautycheck. Ursachen und Folgen von Attraktivität. Regensburg, abgerufen am 09.02.2010 unter http://www.beautycheck.de.

Bryk, Anthony S. / Raudenbush, Stephen W. (1992): Hierarchical Linear Models: Applications and Data Analysis Methods. Newbury Park, London / New Delhi.

Budesheim, Thomas L. / DePaula, Stephen J. (1994): Beauty or the beast? The effects of appearance, personality, and issue information on evaluations of political candidates, in: Personality and Social Psychology Bulletin 20, S. 339-348.

Cash, Thomas F. / Gillen, Barry / Burns, D. Steven (1977 a): Sexism and "beautyism" in personnel consultant decision making, in: Journal of Applied Psychology 62, S. 301-310.

Cash, Thomas F. / Kehr, Jo A. / Polyson, James / Freeman, Valerie (1977 b): The role of physical attractiveness in peer attribution of psychological disturbance, in: Journal of Consulting and Clinical Psychology 45, S. 987-993.

Clifford, Margarete M. / Walster, Elaine (1973): The effect of physical attractiveness on teacher expectations, in: Sociology of Education 46, S. 248-256.

Crouch, Alanda / Degelman, Douglas (1998): Influence of female body images in printed advertising on self-ratings of physical attractiveness by adolescent girls, in: Perceptual and Motor Skills 87, S. 1-2.

Cunningham, Michael R. (1986): Measuring the Physical in Physical Attractiveness: Quasi-Experiments on the Sociobiology of Female Beauty, in: Journal of Personality and Social Psychology 50, S. 925-935. 
Cunningham, Michael R. / Barbee, Anita P. / Pike, Carolyn L. (1990): What Do Women Want? Facialmetric Assessment of Multiple Motives in the Perception of Male Physical Attractiveness, in: Journal of Personality and Social Psychology 59, S. 61-72.

Cunningham, Michael R. / Roberts, Alan R. / Barbee, Anita P. / Druen, Perri B. / Wu, Cheng-Huan (1995): „Their ideas of beauty are, on the whole, the same as ours": Consistency and Variability in the CrossCultural Perception of Female Physical Attractiveness, in: Journal of Personality and Social Psychology 68, S. 261-279.

Dabbs, James M. / Stokes, Neil A. III (1975): Beauty is Power: The Use of Space on the Sidewalk, in: Sociometry 38, S. 551-557.

Dermer, Marshall / Thiel, Darrel L. (1975): When beauty may fail, in: Journal of Personality and Social Psychology 31, S. 1168-1176.

Deseran, Forrest A. / Chung, Chang-Soo (1979): Appearance, Role-Taking, and Reactions to Deviance: Some Experimental Findings, in: Social Psychology Quarterly 42, S. 426-430.

DeShields, Oscar W. / Kara, Ali / Kaynak, Erdener (1996): Source effects in purchase decisions: the impact of physical attractiveness and accent of salespersons, in: International Journal of Research in Marketing 13, S. 89-101.

Dion, Karen K. / Berscheid, Ellen (1974): Physical attractiveness and peer perception among children, in: Sociometry 37, S. 1-12.

Dion, Karen K. / Berscheid, Ellen / Walster, Elaine (1972): What is Beautiful is Good in: Journal of Personality and Society Psychology 24, S. 285-290.

Downs, A. Chris / Lyons, Phillip M. (1991): Natural observances of the links between attractiveness and initial legal judgements, in: Personality and Social Psychology 10, S. 418-421.

Eagly, Alice H. / Ashmore, Richard D. / Makhijani, Mona G. / Longo, Laura C. (1991): What is beautiful is good, but ...: A meta-analytic review of research on the physical attractiveness stereotype, in: Psychological Bulletin 110, S. 109-128.

Efran, Michael G. (1974): The Effect of Physical Appearance on the Judgment of Guilt, Interpersonal Attraction, and Severity of Recommended Punishment in Simulated Jury Task, in: Journal of Research in Personality 8, S. 45-54.

Elder, Glen H. (1969): Appearance and Education in Marriage Mobility, in: American Sociological Review 34, S. 519-533.

Esser, Hartmut (1991): Der Doppelpaß als soziales System, in: Zeitschrift für Soziologie 20, S. 153-166.

Esses, Victoria M. / Webster, Christopher D. (1988): Physical attractiveness, dangerousness, and the Canadian criminal code, in: Journal of Applied Social Psychology 18, S. 1017-1031.

Feingold, Alan (1988): Matching for attractiveness in romantic partners and same-sex friends: A metaanalysis and theoretical critique, in: Psychological Bulletin 104, S. 226-235.

Feingold, Alan (1992): Good-looking people are not what we think, in: Psychological Bulletin 111, S. 304-341.

Friedman, Heidi / Zebrowitz, Leslie A. (1992): The Contribution of Typical Sex Differences in Facial Maturity to Sex Role Stereotypes, in: Personality and Social Psychology Bulletin 18, S. 430-438.

Gangestad, Steven W. / Thornhill, Randy (1997): The evolutionary psychology of extra-pair sex: The role of fluctuating asymmetry, in: Evolution and Human Behavior 18, S. 69-88.

Gillen, Barry (1981): Physical Attractiveness: A Determinant of Two Types of Goodness, in: Personality and Social Psychology Bulletin 7, S. 277-281.

Grammer, Karl (2002): Signale der Liebe. Die biologischen Gesetze der Partnerschaft, Frankfurt / M.

Grammer, Karl / Fink, Bernhard / Møller, Anders P. / Thornhill, Randy (2003): Darwinian aesthetics: sexual selection and the biology of beauty, in: Biological Review 78, S. 385-407. 
Guerra, Daniel N. (1998): The influence of female clients' physical attractiveness on college students' and psychotherapists' judgement of mental health. Dissertation Abstract International: Section B: The science and engineering 1998-58, S. 3966.

Hamermesh, Daniel S. / Biddle, Jeff E. (1994): Beauty and the Labor Market, in: American Economic Review 84, S. 1174-1194.

Hanson Frieze, Irene / Olson, Josephine, E. / Russel, June (1991): Attractiveness and income for men and women in management, in: Journal of Applied Social Psychology 21, S. 1039-1057.

Harper, Barry (2000): Beauty, Stature and the Labour Market: A British Cohort Study, in: Oxford Bulletin of Economics and Statistics 62, S. 771-800.

Hartnett, John J. / Balley, Kent O. / Hartley, Craig S. (1974): Body height, position and sex as determinants of personal space, in: Journal of Psychology 87, S. 129-136.

Hatfield, Elaine / Sprecher, Susan (1986): Mirror, Mirror: The Importance of Looks in Everyday Life. Albany NY.

Heilman, Madeline E. / Saruwatari, Lois R. (1979): When Beauty Is Beastly: The Effects of Appearance and Sex on Evaluation of Job Applicants for Managerial and Nonmanagerial Jobs, in: Organizational Behavior and Human Performance 23, S. 360-372.

Henss, Ronald (1987): Zur Beurteilerübereinstimmung bei der Einschätzung der physischen Attraktivität junger und alter Menschen, in: Zeitschrift für Sozialpsychologie 18, S. 118-130.

Henss, Ronald (1992): „Spieglein, Spieglein an der Wand ...“. Geschlecht, Alter und physische Attraktivität, Weinheim.

Hosoda, Megumi / Stone-Romero, Eugene F. / Coats, Gwen (2003): The Effects of Physical Attractiveness on Job-Related Outcomes: A Meta-Analysis of Experimental Studies, in: Personnel Psychology 56, S. 431-462.

Hox, Joop J. (2002): Multilevel Analysis. Techniques and Applications, New Jersey / London.

Iliffe, Alan H. (1960): A study of preferences in feminine beauty, in: British Journal of Psychology 51, S. 267-273.

Kalter, Frank (1999): Ethnische Kundenpräferenzen im deutschen Sport? Der Fall der Fußballbundesliga, in: Zeitschrift für Soziologie 28, S. 219-234.

Kalter, Frank (2005): Reduziert Wettbewerb tatsächlich Diskriminierungen? Eine Analyse der Situation von Migranten im Ligensystem des deutschen Fußballs, in: Sport und Gesellschaft - Sport and Society 2, S. 1-39.

Kanazawa, Satoshi / Still, Mary C. (2000): Teaching may be hazardous to your marriage, in: Evolution and Human Behaviour 21, S. 185-190.

Klein, Markus / Rosar, Ulrich (2006): Das Auge hört mit! Der Einfluss der physischen Attraktivität des Lehrpersonals auf die studentische Evaluation von Lehrveranstaltungen - eine empirische Analyse am Beispiel der Wirtschafts- und Sozialwissenschaftlichen Fakultät der Universität zu Köln, in: Zeitschrift für Soziologie 35, S. 305-316.

Klein, Markus / Rosar, Ulrich (2007): Ist Deutschland reif für eine Kanzlerin? Eine experimentelle Untersuchung aus Anlass der Bundestagswahl 2005, in: Frank Brettschneider / Oskar Niedermayer / Barbara Pfetsch / Bernhard Wessels (Hrsg.): Die Bundestagswahl 2005. Analysen aus Sicht der Wahlforschung, der Kommunikationswissenschaft und der Parteienforschung, Wiesbaden, S. 271-291.

Klein, Markus / Rosar, Ulrich (2009): Sie, Sie, Sie oder Er? Angela Merkel im Spiegel der Daten einer experimentellen Befragung, in: Oscar W. Gabriel / Jürgen W. Falter / Bernhard Wessels (Hrsg.): Wahlen und Wähler. Analysen aus Anlass der Bundestagswahl 2005, Wiesbaden, S. 346-357.

Köhler, Bernd (1984): Physische Attraktivität und Persönlichkeitsmerkmale, in: Manfred Amelang / HansJoachim Ahrens (Hrsg.): Brennpunkte der Persönlichkeitsforschung, Band 1. Göttingen, S. 139-153.

Krantz, Murray (1987): Physical attractiveness and popularity: A predictive study, in: Psychological Reports 60, S. $723-726$. 
Landy, David / Sigall, Harold (1974): Beauty is talent: Task evaluation as a function of the performer's physical attractiveness, in: Journal of Personality and Social Psychology 29, S. 299-304.

Langlois, Judith H. / Kalakanis, Lisa / Rubenstein, Adam J. / Larson, Andrea / Hallam, Monica / Smoot, Monica (2000): Maxims or myths of beauty? A meta-analytic and theoretical review, in: Psychological Bulletin 126, S. 390-423.

Langlois, Judith H. / Ritter, Jean M. / Casey, Rita J. / Sawin, Douglas B. (1995): Infant attractiveness predicts maternal behaviors and attitudes, in: Developmental Psychology 31, S. 464-472.

Mace, Kenneth C. (1972): The "overt-bluff" shoplifter: who gets caught?, in: Journal of Forensic Psychology 4, S. 26-30.

Maisonneuve, Jean / Bruchon-Schweitzer, Marilou (1999): Le corps et la beauté, Paris.

Maner, Jon K. / Kenrick, Douglas T. / Becker, Vaughn D. / Delton, Andreas W. / Hofer, Brian / Wilbur, Chris J. / Neuberg, Steven L. (2003): Sexually Selective Cognition: Beauty Captures the Mind of the Beholder, in: Journal of Personality and Social Psychology 85, S. 1107-1120.

Marwick, Arthur (1988): Beauty in History. Society, politics and personal appearance c. 1500 to the present, London.

Mazur, Allan / Mazur, Julie / Keating, Caroline (1984): Military Rank Attainment of a West Point Class: Effects of Cadets Physicals Features, in: American Journal of Sociology 90, S. 125-150.

McCabe, Viki (1988): Facial proportions, perceived age, and caregiving, in: Thomas R. Alley (Hrsg.): Social and Applied Aspects of Perceiving Faces. Hillsdale, S. 89-95.

Miller, Arthur G. (1970): Role of physical attractiveness in impression formation, in: Psychonomic Science 19, S. 241-243.

Mueller, Ulrich / Mazur, Allan (1996): Facial Dominance of West Point Cadets as a Predictor of Later Military Rank, in: Social Forces 74, S. 823-850.

Mulford, Matthew / Orbell, John / Shatto, Catherine / Stockard, Jean (1998): Physical Attractiveness, Opportunity and Success in Everyday Exchange, in: American Journal of Sociology 103, S. 1565-1593.

Patzer, Gordon L. (1985): The Physical Attractiveness Phenomena, New York.

Podratz, Kenneth E. / Dipboye, Robert L. (2002): In Search of the „Beauty is Beastly“ Effect. Paper presented at the $17^{\text {th }}$ Annual Conference of the Society for Industrial and Organizational Psychology, April 2002, Toronto.

Reingen, Peter H. / Kernan, Jerome B. (1993): Social Perception and Interpersonal Influence: Some Consequences of the Physical Attractiveness Stereotype in a Personal Selling Setting, in: Journal of Consumer Psychology 2, S. 25-38.

Renz, Ulrich, 2006: Schönheit - Eine Wissenschaft für sich, Berlin.

Ritter, Jean M. / Casey, Rita J. / Langlois, Judith H. (1991): Adults' responses to infants varying in appearance of age and attractiveness, in: Child Development 62, S. 68-82.

Rosar, Ulrich (2009): Fabulous Front-Runners. Eine Analyse zur Bedeutung der physischen Attraktivität von Spitzenkandidaten für den Wahlerfolg ihrer Parteien und zu den Möglichkeiten der Gegensteuerung durch das Wahlrecht, in: Politische Vierteljahresschrift 50, S. 754-773.

Rosar, Ulrich / Hagenah, Jörg / Klein, Markus (2010): Elf Dressmen sollt ihr sein. Eine Untersuchung zum Zusammenhang zwischen physischer Attraktivität und den Aussichten auf ökonomischen Erfolg im Profifußball, Köln / Hannover (unveröffentlichtes Manuskript).

Rosar, Ulrich / Klein, Markus (2009): Mein(schöner)Prof.de. Die physische Attraktivität des akademischen Lehrpersonals und ihr Einfluss auf die Ergebnisse studentischer Lehrevaluationen, in: Kölner Zeitschrift für Soziologie und Sozialpsychologie 61, S. 621-645.

Rosar, Ulrich / Klein, Markus / Beckers, Tilo (2008): The frog pond beauty contest. Physical attractiveness and electoral success of the constituency candidates at the North Rhine-Westphalia state election of 2005, in: European Journal of Political Research 47, S. 64-79. 
Rosenblum, Gianine D. / Lewis, Michael (1999): The relations among body image, physical attractiveness, and body mass in adolescence, in: Child Development 70, S.50-64.

Ross, Michael B. / Salvia, John (1975): Attractiveness as a biasing factor in teacher judgments, in: American Journal of Mental Deficiency 80, S. 96-98.

Rost, Detlef H. (1993): Attraktive Grundschulkinder. In: Manfred Hassebrauck / Reiner Niketta (Hrsg.): Physische Attraktivität. Göttingen, S. 271-306.

Savin-Williams, Ritch C. (1979): Dominance hierarchies in groups of early adolescents, in: Child Development 50, S. 923-935.

Sczesny, Sabine (2003): A closer look beneath the surface: Various facets of the think-manager-thinkmale stereotype, in: Sex Roles 49, S. 353-363.

Shackelford, Todd. K. / Weekes-Shackelford, Viviana A. / LeBlanc, Gregory J. / Bleske, April L. / Euler, Harald A. / Hoier, Sabine (2000): Female coital orgasm and male attractiveness, in: Human Nature 11, S. 299-306.

Skarupke, Robert (2000): Quantifizierung des Heimvorteils im deutschen Profifußball: Eine empirische Untersuchung für die 1. Fußball-Bundesliga, Arbeitspapier Nr. 20 des Instituts für Statistik und Ökonometrie, Mainz.

Snijders, Tom / Bosker, Roel (1999): Multilevel Analysis. An introduction to basic and advanced multilevel modeling. London, Thousand Oaks und New Dehli.

Solnick, Sara J. / Schweitzer, Maurice E. (1999): The Influence of Physical Attractiveness and Gender on Ultimatum Game Decisions, in: Organizational Behavior and Human Decision Processes 79, S. 199-215.

Steffensmeier, Darrell J. / Terry, Robert M. (1973): Deviance and Respectability: An Observational Study of Reactions to Shoplifting, in: Social Forces 51, S. 417-426.

Thornhill, Randy / Gangestad, Steven W. (1994): Human fluctuating asymmetry and sexual behaviour, in: Psychological Science 5, S. 297-302.

Thornhill, Randy / Gangestad, Steven W. / Comer, Randall (1995): Human Female Orgasm and Male Fluctuating Asymmetry, in: Animal Behavior 50, S. 1601-1615.

Turner, Sherry L. / Hamilton, Heather / Jacobs, Meija / Angood, Laurie M. / Hovde Dwyer, Deanne (1997): The influence of fashion magazines on the body image satisfaction of college women: An exploratory analysis, in: Adolescence 32, S. 603-614.

Udry, J. Richard / Eckland, Bruce K. (1984): The benefits of being attractive: Differential payoffs for men and women, in: Psychological Reports 54, S. 47-56.

Vaughn, Brian E. / Langlois, Judith H. (1983): Physical attractiveness as a correlate of peer status and social competence in preschool children, in: Developmental Psychology 19, S. 561-567.

Waller, Willard W. (1937): The rating and dating complex. American Sociological Review 2, S. 727-734.

Walster, Elaine / Aronson, Vera / Abrahams, Darcy / Rottmann, Leon (1966): Importance of Physical Attractiveness in Dating Behavior, in: Journal of Personality and Social Psychology 4, S. 508-516.

White, Gregory L. (1980): Physical attractiveness and courtship progress, in: Journal of Personality and Social Psychology 39, S. 660-668.

White Stephan, Cookie / Langlois, Judith H. (1984): Baby beautiful: Adult attributions of infant competence as a function of infant attractiveness, in: Child Development 55, S. 576-585.

PD Dr. Ulrich Rosar

Dr. Jörg Hagenah

Forschungsinstitut für Soziologie der Universität zu Köln

Greinstraße 2

50939 Köln

rosar@wiso.uni-koeln.de

hagenah@wiso.uni-koeln.de
Prof. Dr. Markus Klein Institut für Politische Wissenschaft der Leibniz Universität Hannover Schneiderberg 50 30167 Hannover m.klein@ipw.uni-hannover.de 